\title{
ESTRUCTURA DE UNA POLÍTICA PÚBLICA PARA LA EMPRESA FAMILIAR DE HONDURAS
}

ISSN 2219-6722

ISSNE 2222-2707

RINA PATRICIA MUNGUÍA LÓPEZ, Dirección de Educación Superior Universidad Nacional Autónoma de Honduras (UNAH), Cuidad Universitaria Tel. (504) 98421003; rinam302@gmail.com

\section{RESUMEN}

En el mundo, se encuentran las denominadas empresas familiares, las cuales son parte de los actores en la dinámica empresarial, el contenido de este trabajo inicia con el abordaje de la experiencia internacional identificando las iniciativas de cada país como punto de partida para el proceso de la investigación.

A pesar de la relevancia de las empresas familiares como generadoras de la estabilidad económica y social de los países, los hallazgos encontrados detectan una similitud: falta políticas públicas que permitan el fomento, mejoramiento y continuidad de las mismas en el tiempo.

Palabras clave: Empresa familiar, teoría de sistemas, emprendedor, política pública. 


\title{
STRUCTURE OF A PUBLIC POLICY FOR FAMILY BUSINESSES OF HONDURAS
}

ISSN 2219-6722

ISSNE 2222-2707

RINA PATRICIA MUNGUÍA LÓPEZ, Dirección de Educación Superior Universidad Nacional Autónoma de Honduras (UNAH), Cuidad Universitaria Tel. (504) 98421003; rinam302@gmail.com

\begin{abstract}
In the world, well known family businesses can be found, which compose part of the actors in the business dynamic. The content of this work begins with the approach of the international experience identifying the initiatives of each country as the starting point for the research process. Despite the relevance of family businesses as generators of economic and social stability of countries, the findings detect a similarity: Public policies are needed to enable the development, improvement and continuity of the same over time.
\end{abstract}

Keywords: Family business, systems theory, entrepreneur, public policy. 


\section{INTRODUCCIÓN}

La temática de empresas familiares (EF), ha sido abordada desde diversas perspectivas; la evidencia estadística señala que su relevancia radica no sólo en una oportunidad para un emprendedor desde el punto de vista mercantil de forma aislada, sino por el contrario, se contrasta con el aporte significativo a nivel económico y social, ya que emplean a 100 millones de personas, esto representa $60 \%$ de las empresas a nivel mundial. (Betancourt; Arcos, Torres \& Olivares Bazán, 2015). Debido a la relevancia plasmada, es necesario prestar interés nacional ante la problemática planteada en el apartado de antecedentes empíricos.

\section{MARCO TEÓRICO / CONCEPTUAL / REFERENCIAL}

\subsection{Ciclos de las empresas familiares}

Puntualiza Dodero (2008), que es necesario analizar el ciclo puesto que es una herramienta para la empresa familiar, sobre todo porque puede servir como un proceso de planeación preventiva para cada uno de sus ciclos.

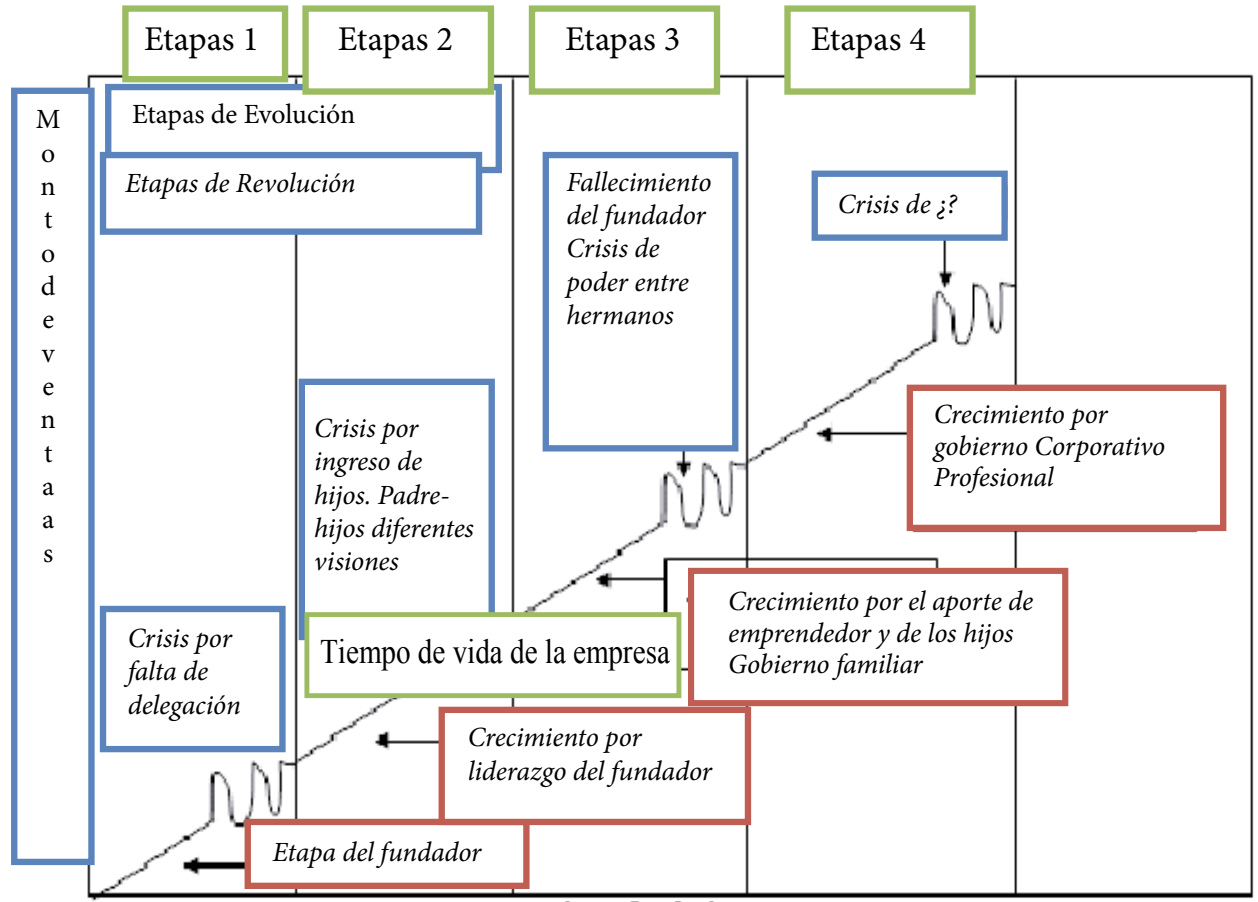

Tiempo de vida de la empresa

Figura No. 3. Ciclos manifestados en las empresas familiares. Fuente: Santiago Dodero, El ciclo de vida de las empresas familiares, Temas de managment, Pág. 9, 2008. 


\subsection{Política pública}

Para Ruiz \& Cadénas, (2015) definen la política pública como la forma de gobernar los entes pertinentes al Estado, desde el punto de vista administrativo, sobre todo en la forma de velar por sus intereses.

Es histórico que las políticas públicas por tanto, provocan los escenarios requeridos para que los efectos transformacionales que el contexto requiere.

Estudios que reflejan la importancia de este tipo de emprendedurismo en diversos países:

\section{TABLA No. 1. Importancia del Emprendedurismo en otros países}

\begin{tabular}{|l|l|l|}
\hline Lugar & Porcentaje de aporte al PIB & Estadística de sucesión \\
\hline España & $50 \%$ (Amat, 2004) & $\begin{array}{l}\text { Martínez Barroso, (2014) } \\
\text { señala que el 25\% llega a la } \\
\text { segunda generación, pero } \\
\text { solamente 9\% a la tercera }\end{array}$ \\
\hline Estados Unidos & $40 \%$ & $\begin{array}{l}66 \% \text { llega a segunda generación } \\
\text { y solo el 12\% sobrevive } \\
\text { (Echezárraga, 2004) }\end{array}$ \\
\hline México & $\begin{array}{l}52 \% \text { son Pymes, de las cuales } \\
65 \% \text { son familiares(Hernández, } \\
2012)\end{array}$ & $\begin{array}{l}45 \% \text { pasa a la segunda } \\
\text { generación y apenas el 20\% } \\
\text { llega a la tercera(KPMG, 2008) }\end{array}$ \\
\hline Colombia & $45 \%$ y 70\% (Economía, 2014) & $\begin{array}{l}\text { 63\% segunda generación; 30\% } \\
\text { tercera (Valda, 2010) }\end{array}$ \\
\hline Chile & $50 \%-75 \%$ (Martínez, 2013) & $\begin{array}{l}\text { 30\% continúa hacia la segunda } \\
\text { generación, apenas 1/3 logra } \\
\text { sobrevivir a la tercera (BCG, } \\
2011)\end{array}$ \\
\hline Argentina & $\begin{array}{l}30 \% \text { segunda y 15\% } \\
\text { respectivamente (IAEF, 2014) }\end{array}$ \\
\hline Honduras & $60 \%$ (familiares, 2015) & $\begin{array}{l}\text { De acuerdo al BID y Cohep, } \\
\text { (2011) el 14\% de las EF llegan } \\
\text { a la segunda generación y } \\
\text { solamente 10\% a la tercera. }\end{array}$ \\
\hline
\end{tabular}

Fuente: Elaborado por la autora, a partir de diferentes fuentes.

Obsérvese que el mayor porcentaje de empresas familiares se encuentran en Chile, seguido de Colombia, Argentina y México respectivamente. En complemento España, históricamente toman su protagonismo las EF como objeto de estudio desde 1983, donde según Amat, (2004) ya empleaban un 65\% de la población económicamente activa (PEA). El mismo autor rescata que en Estados Unidos el caso es similar al país anterior, ya que generan ocupaciones para el $40 \%$ de la PEA.

La tasa de mortalidad de las empresas familiares es cada vez más alarmante, se resume que los países con mayor porcentaje de sobreviviencia en la segunda y tercera generación de estas empresas son Estados Unidos y Colombia respectivamente. España, Chile y Hon- 
duras se encuentran con datos muy similares en la tercera generación, lo anterior requiere dar un vistazo a cada país de forma puntual para observar las prácticas de manejo dirigida a las EF.

En las naciones señaladas existen programas especiales dirigidos al apoyo y conservación de las empresas familiares, por su alto grado de protagonismo como entes del impulso empresarial, se presentan seguidamente detalle de los países analizados, debido al aporte significativo que tienen las EF en el Producto Interno Bruto.

Chile, uno de los países con el mayor aporte del PIB de las EF, ha tomado como punto de partida la educación, herramienta que permite potenciar los talentos y asegurar su continuidad a través de sucesiones exitosas. Las universidades han tenido la iniciativa de plantear a nivel académico y empresarial soluciones que apoyan la continuidad generacional, algunas han pasado a formar parte de redes importantes como The Family Businness Network (La Red de Familias Empresarias), donde se realizan actividades que generan una base sólida de la transformación, profesionalización y trascendencia de las buenas prácticas empresariales.

Para el caso de Colombia, cuenta a nivel de nacional con firmas consultoras dedicadas a asesorar las EF, caso similar a Chile, donde también las universidades han sido actores relevantes para su sobrevivencia, buenas prácticas que permiten dinamizar y balancear los dos sistemas Familia y Empresa. (Vélez, Holguín, De la Hoz, Durán, \& Gutiérrez, 2008). Históricamente España de acuerdo a Arieu, (2012) cuenta con una de las más prestigiosas organizaciones conformadas por distintas empresas a nivel nacional denominada: la Asociación de Empresas Familiares de Castilla - La Mancha, originaria de Salamanca, sitio que promueve la longevidad y la cultura empresarial. A pesar de la notabilidad de dicha institución, el gobierno solamente toma en cuenta sus opiniones más no suscita las oportunidades de inclusión significativas que generen políticas públicas adecuadas en pro del desarrollo empresarial familiar.

Estados Unidos, cuenta con escuelas de negocios como Harvard y Babson, ambas mantienen programas de formación orientado a la gestión de empresas familiares. Al igual que Chile, también se encuentra en la red de The Family Businness Network y Family Enterprise USA (FEUSA), la última realiza encuestas de las políticas de gobernanza a lo interno de las familias empresarias, que sirven como referencias para determinar sus prácticas de gestión administrativa y familiar.

En Argentina, la prestigiosa escuela de negocios Aden cuenta con el Instituto Latinoamericano de la Empresa Familiar, ente que genera espacios de convergencia, con metodologías innovadoras a través de herramientas de fortalecimiento a lo interno de las EF. En complemento la ciudad de Córdoba, es única en la creación del Centro Regional de Apoyo y Desarrollo para la Pequeña Empresa como un apoyo por parte de la municipalidad de esa localidad (Córdoba, 2002). Dicho lugar tiene como eje fundamental el soporte de las microempresas con énfasis en la educación y la integración familiar ya que el talón de Aquiles que conlleva al cierre de los negocios familiares son precisamente los abordados por el centro. La falta de educación y formación básica que permita la continuidad del negocio, es una de las deficiencias de las EF, entiéndase que también parte de esa columna 
proviene del hogar, siendo la familia origen de la continuidad empresarial.

Lo anterior asume dos premisas: primera si la familia no es propiamente conducida y termina en la división emocional, esa disrupción se verá reflejado en la empresa, ya que la repartición de bienes provocará falencias que afectan la continuidad en el transcurso del tiempo.

Segunda, la familia tiene la responsabilidad de orientar a sus sucesores en la formación profesional que permita el desarrollo y crecimiento empresarial, de no suceder dicha expectativa la sucesión a generaciones subsecuentes no sucederá, por la falta de liderazgo y visión a largo plazo.

En pocas palabras, en la familia se adquiere el apego emocional y formación de valores hacia la empresa, al no existir esa conexión causada por los escenarios presentados, la discontinuidad es casi un hecho, situación que no solamente crea un impacto a nivel micro sino macro representado en el PIB y social con respecto a la generación de empleos; en resumen el bienestar de la EF es también la prosperidad para cualquier nación.En complemento, México toma la delantera siendo más proactivo, eleva el nivel de importancia de las EF creando un marco legal a nivel nacional denominado: Estrategia integral de políticas públicas para favorecer el sano desenvolvimiento de los negocios familiares, desde ese momento denota que debe propiciar los esfuerzos a una eficaz política de Estado. (Secretaría de Economía México, 2015)

Es una estrategia híbrida, donde los entes gubernamentales tienen como objetivo brindar el soporte requerido para el desarrollo de las EF y los privados otorgando el financiamiento propio para las mismas con acciones puntuales. La política pública nace a partir de la comprensión económica y social ya que representa la tercera parte de la PEA es sostenida por este tipo de emprendimientos.

La primera y única iniciativa en Honduras el 90\% de las Pymes son familiares, uno de los principales esfuerzos para su conservación fue realizada con una alianza entre el Consejo Hondureño de la Empresa Privada (COHEP) y el Banco Interamericano de Desarrollo (BID), procurando el fortalecimiento de forma puntual a través de protocolos para la EF, donde se encaminan a una mejor gestión y efectiva sucesión, (Cohep, 2009).

No obstante al interpretar la referida cantidad de pymes familiares hondureñas, no basta con realizar solo un programa de fortalecimiento para la continuidad empresarial de esta índole, se requieren esfuerzos públicos y privados permanentes que permitan el crecimiento y no el detrimento de la misma, que por tanto aqueja la frágil economía del país. Por esas razones se propone generar una política pública para la empresa familiar hondureña, que tenga como ejes transversales: el fomento, profesionalización, permanencia, unidad, financiamiento y continuidad de la misma.

\section{METODOLOGÍA}

El diseño de esta investigación es exploratorio, ya que solamente se toman en cuenta los insumos bibliográficos de segunda y tercera mano para la elaboración de la propuesta en cuestión. (Hernández Sampieri, Fernández Collado, \& Baptista Lucio, 2010) 


\section{IMPLICACIONES PRÁCTICAS}

Se considera los mínimos aspectos que la propuesta debe contener:

a) Elaborar un diagnóstico actualizado de las necesidades del país con respecto a la problemática de la empresa familiar.

b) Comprender la relevancia del papel de las empresas familiares en la contribución económica, social y emocional a nivel nacional, con indicadores relevantes al respecto.

c) Creación del marco jurídico enfocado a la protección en cada una de las etapas descritas, sobre todo en los puntos de identificados de urgencia en el diagnóstico.

d) Elevar a nivel de ley el marco jurídico contemplado anteriormente.

e) Identificar los órganos adecuados para el manejo práctico de la política que garantice la continuidad de la empresa familiar, apoyado de una triple hélice: empresa familiar, gobierno y academia.

f) Crear programas que eleven competitividad, creando así un efecto dominó en los indicadores macroeconómicos.

Los beneficios de la implementación práctica de esta política va en doble vía, la primera privada hacia la EF puntualmente, segunda al gobierno por la contribución macro que significa el mantenimiento de este tipo de emprendedurismo. Se debe adicionar a los enfoques actuales del fomento hacia el empleo, la conservación de la EF para generar un impacto significativo, tal como se ha descrito anteriormente social y económico, manteniendo la salud empresarial dentro del país.

\section{CONCLUSIONES E IMPLICACIONES}

En el transcurso del tiempo las empresas familiares han tomado el lugar que merecen en algunos países, sin embargo las iniciativas aún no son las pertinentes para su despegue y continuidad real.

Al observar la información obtenida de distintas naciones, se encuentran las siguientes similitudes bajo el enfoque de la teoría de los sistemas: la empresa familiar conforma un aporte económico importante en los indicadores principales, seguido del social. México ha comprendido el papel preponderante, por tanto ha desarrollado una estrategia que ayuda a mantener la vitalidad de estas empresas, en resumen el bienestar de la EF es también la prosperidad para cualquier nación. 


\section{REFERENCIAS BIBLIOGRÁFICA}

Alonso Nuez, M. J., \& Galve Górriz, C. (2008). El emprendedor y la empresa una revisión teórica de los determinantes de su constitución. Acciones e Investigaciones sociales, 5 - 44.

Amat, J. (2004). La Continuidad de la empresa Familiar. España: Gestión 2000.

Arieu, A. (25 de Mayo de 2012). http://negociosymanagement.com.ar/?p=466.

Obtenido de http://negociosymanagement.com.ar

BCG. (21 de Octubre de 2011). http://www.economiaynegocios.cl/. Obtenido de $\mathrm{http}: / / \mathrm{www}$. economiaynegocios.cl/noticias/noticias.asp?id=89742

Betancourt Enriquez, Ana Diana; Arcos Moreno, Sergio; Torres Vargas, Alma Elizama ; Olivares Bazán, León Donizetty. (11 de 09 de 2015). Empresas familiares. Obtenido de http://www.eumed.net: http://www.eumed.net/rev/ tlatemoani/09/emvb.html

BID. (3 de Diciembre de 2011). http://www.departamento19.hn. Obtenido de http:// www.departamento 19.hn/index.php/economia/banca/2679-honduras-con-altamortalidad-de-empresas-familiares.html

Blanco, M. E. (2008). Iniciativa emprendedora . España: Editex.

Caldas M. E., Reyes Carrión \& Heras A. (2009). Empresa e Iniciativa Emprendedora. España: Editex.

Cohep. (2009). Familia Empresarial. Recuperado el 23 de Junio de 2010, de https://familiaempresarial.cohep.com/index.php?option=com content\&view $=$ article \&id $=5 \&$ Itemid $=34$

CONGREGADO, E., MILLÁN, J., RAYMONG, J., ROIG, J., SALAS, V., SÁNCHEZ-ASÍN, J., \& SERRANO, L. (2008). El capital humano y los emprendedores. Valencia.

Córdoba, M. d. (01 de Octubre de 2002). Centro Regional de Apoyo y Desarrollo para la Pequeña Empresa. Obtenido de http://habitat.aq.upm.es: http://habitat. aq.upm.es/dubai/02/bp113.html

Echezárraga, J. M. (2004). Desafíos y oportunidades de las empresas familiares. $V$ Encuentro de la pequeña y mediana empresa, (pág. 25). Santiago.

Economía. (02 de Mayo de 2014). http://www.colombia.com. Obtenido de http:// www.colombia.com/actualidad/economia/sdi/87823/las-empresas-familiaresrepresentan-el-70-de-la-industria-colombiana

Economía, S. d. (2015). http://mexico.smetoolkit.org/mexico. Obtenido de http:// mexico.smetoolkit.org/mexico/es/content/es/4341/Programa-de-negocios- 
familiares

familiares, E. (29 de Mayo de 2015). http://www.finanzas.com. Obtenido de http:// www.finanzas.com/noticias/economia/20140529/empresas-familiares-aportanregional-2680585.html

Fuentes, F., \& Sánchez, S. (2010). Análisis del perfil emprendedor: una perspectiva de género. Estudios de economía Aplicada, 1-28.

Hernández Sampieri, R., Fernández Collado, C., \& Baptista Lucio, P. (2010). Metodología de Investigación. México: McGraw Hill.

Hernández, I. V. (28 de Marzo de 2012). http://www.cnnexpansion.com. Obtenido de http://www.cnnexpansion.com/emprendedores/2012/03/12/pymes-el-eje-dela-economia-mexicana

IAEF. (01 de Abril de 2014). http://www.iadef.org. Obtenido de http://www.iadef. org/2014/04/01/que-pasa-en-mi-empresa-familiar/

Johansen, O. (2004). Introducción a la teoría general de sistemas Escrito. México: Limusa.

KPMG. (2008). http://www.mundopymeabm.org.mx/. Obtenido de http://www. mundopymeabm.org.mx/pdf/PlandeSucesionenlasEmpresasFamiliaresKPMG. pdf

Martínez, A. B. (2014). ¿Por qué desaparecen las empresas familiares extremeñas? Geographos, 98 - 132.

Martínez, J. (22 de Julio de 2013). http://mba.americaeconomia.com. Obtenido de http://mba.americaeconomia.com/articulos/entrevistas/juan-arriaga-se-refiereal-valor-de-las-empresas-familiares-de-la-region

Mundial, B. I. (2010). Mujeres Empresarias: Barreras y oportunidades en el Sector privado formal de LA y Caribe. Washington: Ledel SAC.

Neubauer, F., \& Lank, A. (2003). La empresa familiar: cómo dirigirla para que perdure. España: Deusto.

Orham, M., \& Scott, D. (2001). "Why women enter into entrepreneurship: an explanatory. Women in Management Review, 232-47.

Ortíz, M. P. (2008). Introducción a la teoría económica. Caracas: Universidad Católica Andrés Bello.

Romero, M. J. (07 de Agosto de 2014). http://www.fenagh.net/web. Obtenido de http://www.fenagh.net/web/pymes-abarcan-mas-del-40-de-la-economia-encentroamerica/

Ruiz, E., \& Cadénas, C. (11 de Septiembre de 2015). http://www.unla.mx/. Obtenido 
de http://www.unla.mx/iusunla18/reflexion/QUE\%20ES\%20UNA\%20 POLITICA\%20PUBLICA\%20web.htm

Valda, J. C. (04 de Noviembre de 2010). http://www.grandespymes.com.ar. Obtenido de http://www.grandespymes.com.ar/2011/09/18/colombia-el-pais-tiene-511000-empresas-familiares-tienen-alta-participacion-en-el-sector-productivodel-pais/

Vélez Montes, D., Holguín Lagos, H., De La Hoz Pinzón, G., Durán Bobadilla, Y., \& Gutiérrez Ayala, I. (2008). Dinámica de la Empresa Familiar Pyme. Dinámica de la Empresa Familiar Pyme, Estudio exploratorio en Colombia. Colombia: Fundes.

Vélez, D., Holguín, H., De la Hoz, G., Durán, Y., \& Gutiérrez, I. (2008). Dinámica de la Empresa Familiar Estudio exploratorio en Colombia. Colombia: Fundes.

\section{AUTORIZACIÓN Y RENUNCIA}

Los (a) autores facultan a CEAT para publicar el escrito en los procedimientos de la conferencia. CEAT o los editores no son responsables por el contenido y las implicaciones de lo que esta expresado en el escrito." 